\title{
Light-Responsive Metal-Organic-Framework as an Oxidase Mimic for Cellular Glutathione Detection
}

\author{
Yufeng Liu, ${ }^{1}$ Min Zhou, ${ }^{1}$ Wen Cao, ${ }^{1}$ Xiaoyu Wang, ${ }^{1}$ Quan Wang, ${ }^{1}$ Sirong Li, ${ }^{1}$ and Hui Wei ${ }^{1,2, *}$ \\ ${ }^{1}$ Department of Biomedical Engineering, College of Engineering and Applied Sciences, Nanjing National Laboratory of \\ Microstructures, Jiangsu Key Laboratory of Artificial Functional Materials, Nanjing University, Nanjing, Jiangsu, 210093, \\ China. \\ ${ }^{2}$ State Key Laboratory of Analytical Chemistry for Life Science and State Key Laboratory of Coordination Chemistry, \\ School of Chemistry and Chemical Engineering, Collaborative Innovation Center of Chemistry for Life Sciences, Nanjing \\ University, Nanjing, Jiangsu 210023, China. \\ E-mail: weihui@nju.edu.cn; Tel: +86-25-83593272; Fax: +86-25-83594648.
}




\section{Table of Contents}

Scheme S1. Synthesis of the photosensitized (PS) organic linker.

Figure S1. ${ }^{1} \mathrm{H}$ NMR spectrum of the PS linker.

Figure S2. UV-visible absorption spectrum of the PS linker.

Figure S3. TEM images of PSMOF.

Figure S4. SEM images of PSMOF.

Figure S5. (A) The effect of concentration on the oxidase-like catalytic activity of PSMOF. All samples were in $0.1 \mathrm{M}$ acetate buffer ( $\mathrm{pH} 4.0)$ containing TMB $(500 \mu \mathrm{M})$. (B) The effect of irradiation time on the oxidase-like catalytic activity of PSMOF. All samples were in $0.1 \mathrm{M}$ acetate buffer ( $\mathrm{pH} 4.0)$ containing TMB $(500 \mu \mathrm{M})$ and PSMOF $(12.5 \mu \mathrm{g} / \mathrm{mL})$.

Figure S6. (A) UV-visible absorption spectra of the oxidized TMB. Five samples in $500 \mu \mathrm{L}$ acetate buffer $(0.10 \mathrm{M}, \mathrm{pH}$ 4.0) containing PSMOF ( $40 \mu \mathrm{L}, 3 \mathrm{mg} / \mathrm{mL})$ and TMB $(10 \mu \mathrm{L}, 25 \mathrm{mM})$ were irradiated under a compact light for $30 \mathrm{~min}$. (B) Normalized irradiation wavelength-dependent oxidase-mimicking activity of PSMOF.

Figure S7. Effects of light source on the oxidase-mimicking activity of PSMOF.

Figure S8. (A) TEM image of PSMOF after the catalytic reaction. (B) TEM image of PSMOF after 2 days of storage under $0.10 \mathrm{M}$ acetate buffer ( $\mathrm{pH}$ 4.0).

Figure S9. (A) UV-visible absorption spectra of four samples in $0.10 \mathrm{M}$ acetate buffer (pH 4.0) containing ABTS (no light irradiation), PSMOF+ABTS (no light irradiation), ABTS under light irradiation, and PSMOF+ABTS (under light irradiation). Inset: corresponding photograph of the four samples. (B) UV-visible absorption spectra of four samples in $0.10 \mathrm{M}$ acetate buffer ( $\mathrm{pH} 4.0$ ) containing OPD (no light irradiation), PSMOF+OPD (no light irradiation), OPD under light irradiation, and PSMOF+OPD under light irradiation. Inset: corresponding photograph of the four samples.

Figure S10. The effect of nitrogen on the oxidase-like catalytic activity of PSMOF.

Figure S11. UV-visible absorption spectra of TMB after catalytic oxidation with PSMOF in the absence and presence of L-Cys with different concentrations $(0-40 \mu \mathrm{M})$.

Figure S12. Effect of ascorbic acid on the oxidase-mimicking activity of PSMOF.

Figure S13. (A) Calibration curve of the commercial GSH kit in the concentration range from 4 to $66 \mu \mathrm{M}$. (B) GSH detection in different cell densities with the commercial GSH kit.

Table S1. Kinetics parameters of PSMOF nanozymes as well as laccase.

Table S2. Comparison of different methods for GSH detection.

References. 


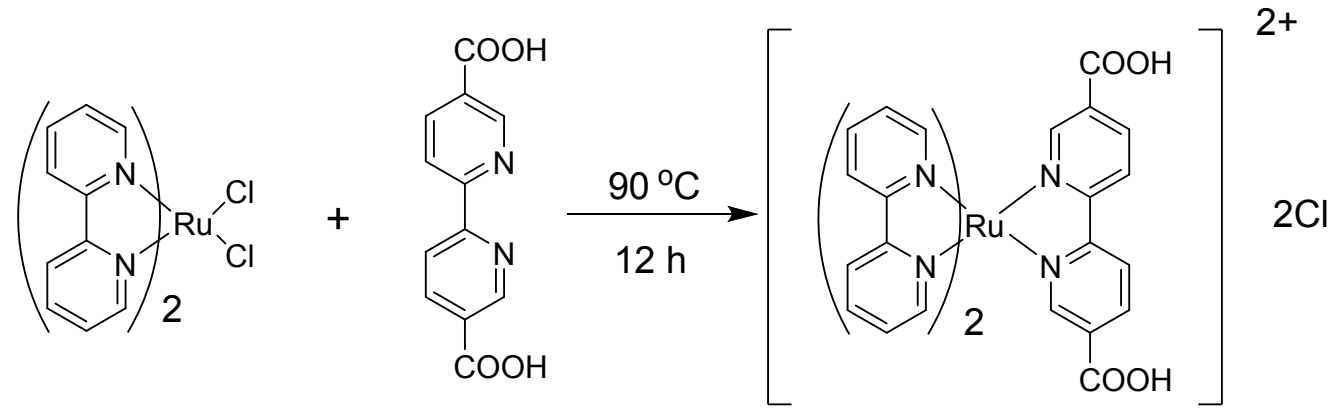

Scheme S1. Synthesis of the photosensitized (PS) organic linker. 


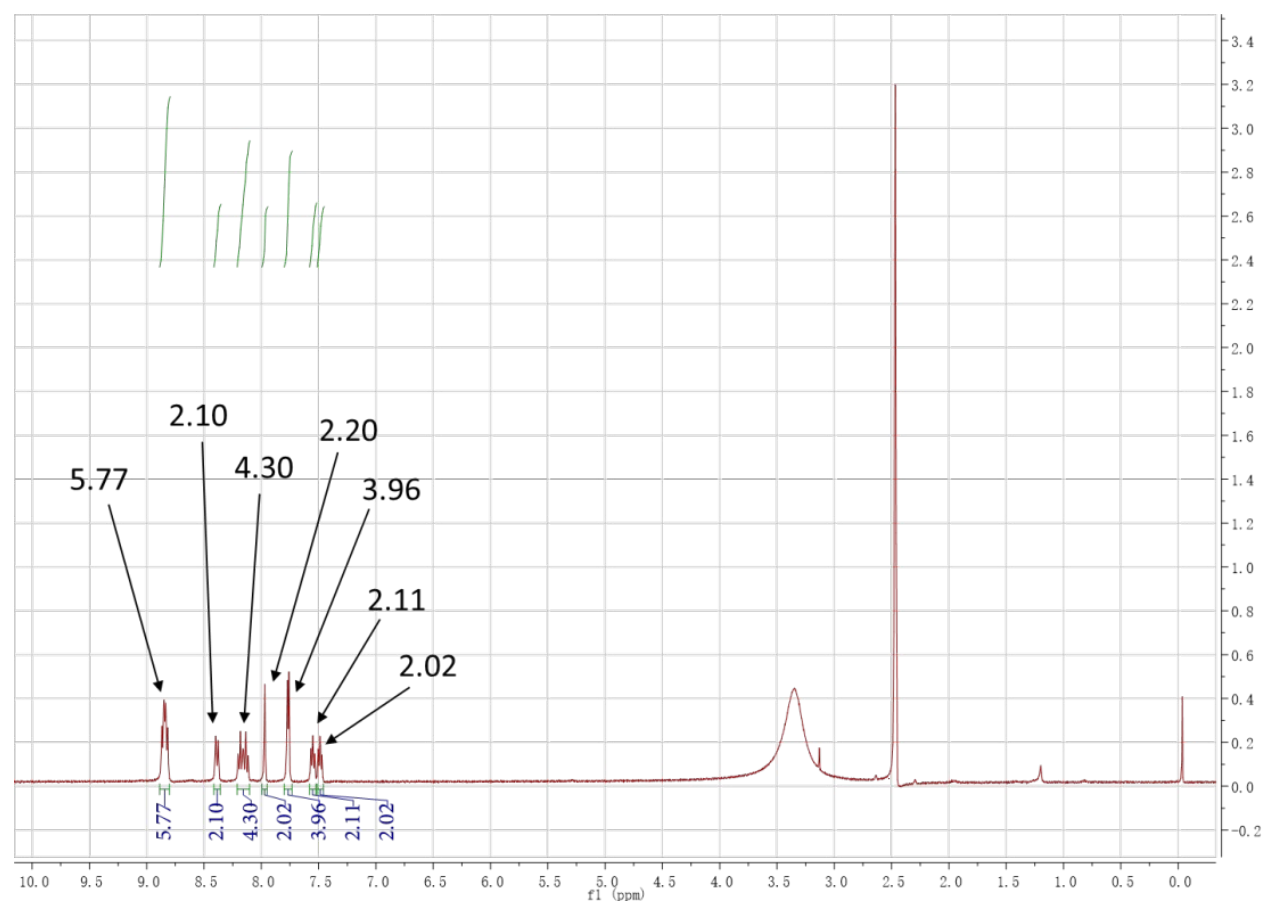

Figure S1. ${ }^{1} \mathrm{H}$ NMR spectrum of the PS linker. 


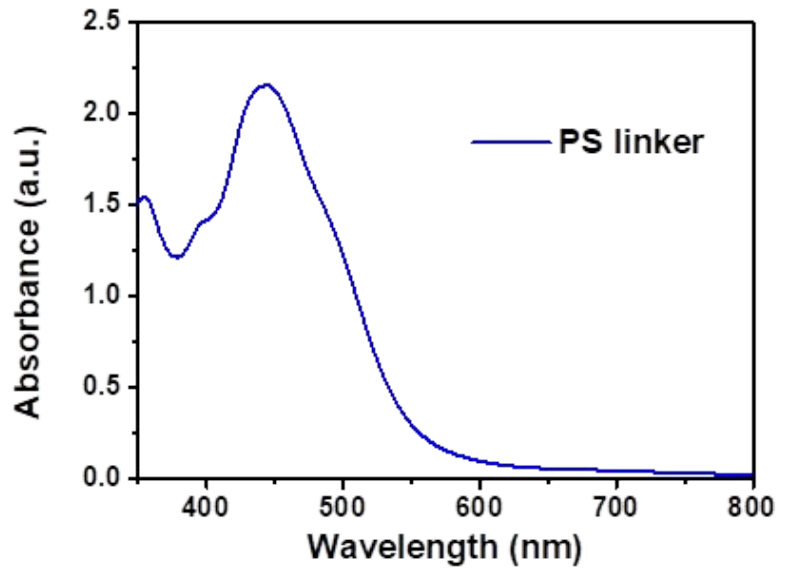

Figure S2. UV-visible absorption spectrum of the PS linker. 
A

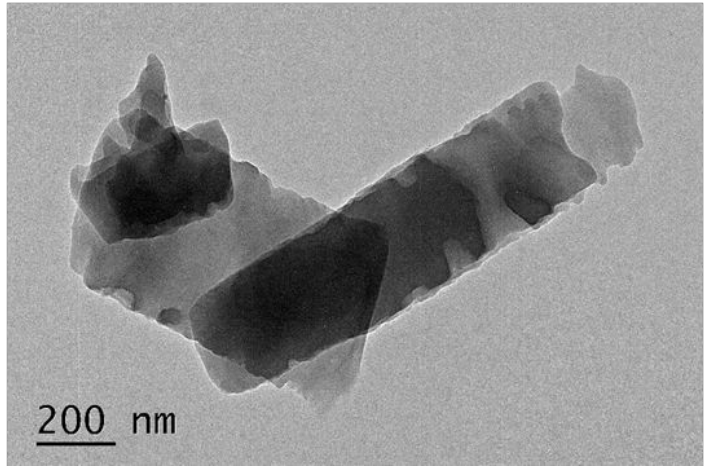

B

$$
\underline{200 \mathrm{~nm}}
$$

Figure S3. TEM images of PSMOF. 
A

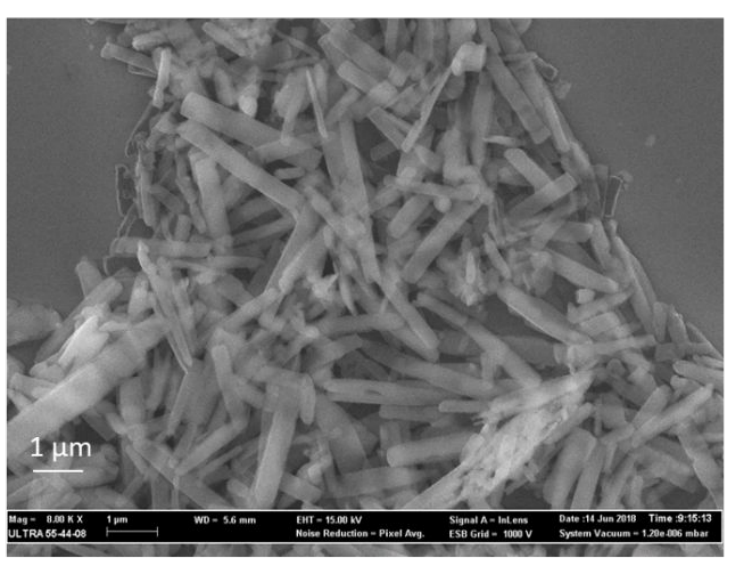

Figure S4. SEM images of PSMOF.

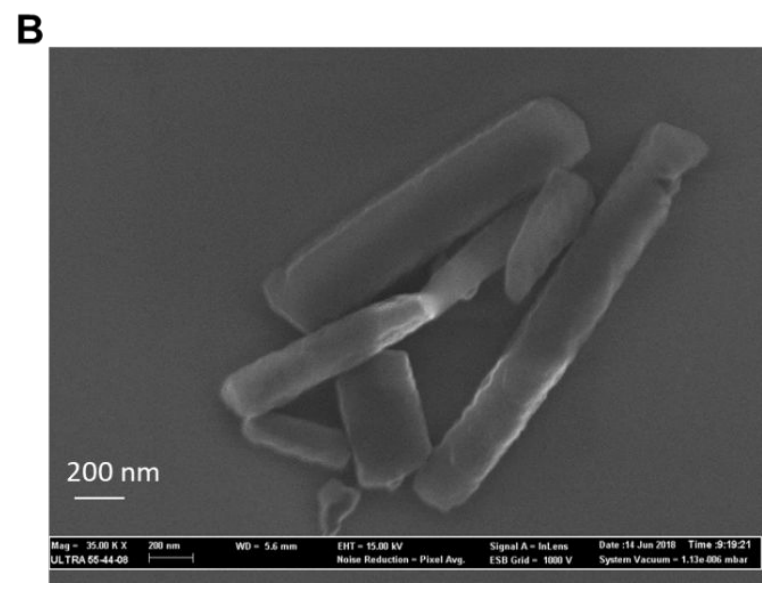


A

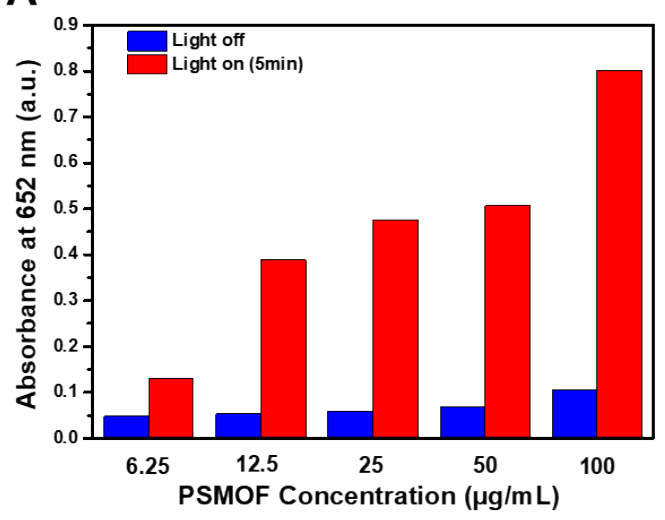

B

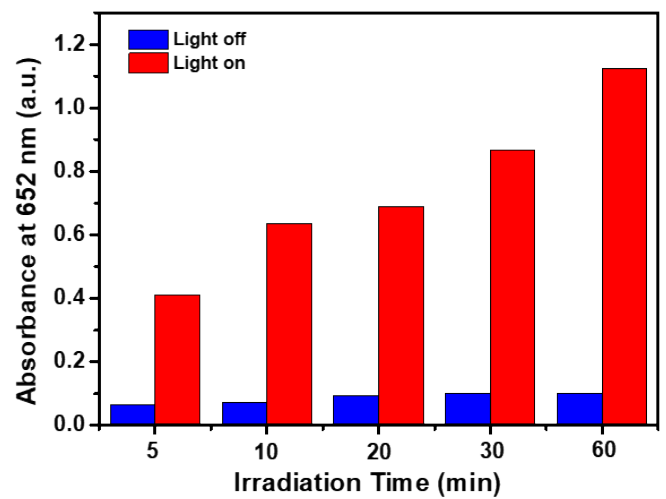

Figure S5. (A) The effect of concentration on the oxidase-like catalytic activity of PSMOF. All samples were in $0.1 \mathrm{M}$ acetate buffer ( $\mathrm{pH} 4.0)$ containing TMB $(500 \mu \mathrm{M})$. (B) The effect of irradiation time on the oxidase-like catalytic activity of PSMOF. All samples were in $0.1 \mathrm{M}$ acetate buffer (pH 4.0) containing TMB (500 $\mu \mathrm{M})$ and PSMOF $(12.5 \mu \mathrm{g} / \mathrm{mL})$. 
A

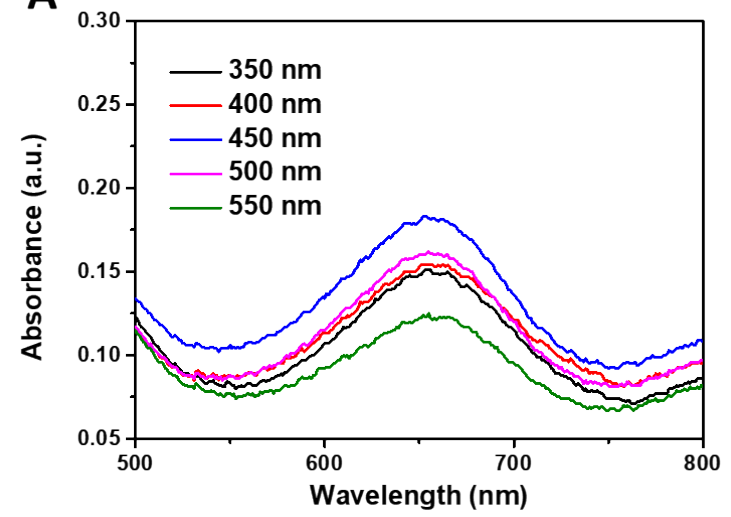

B

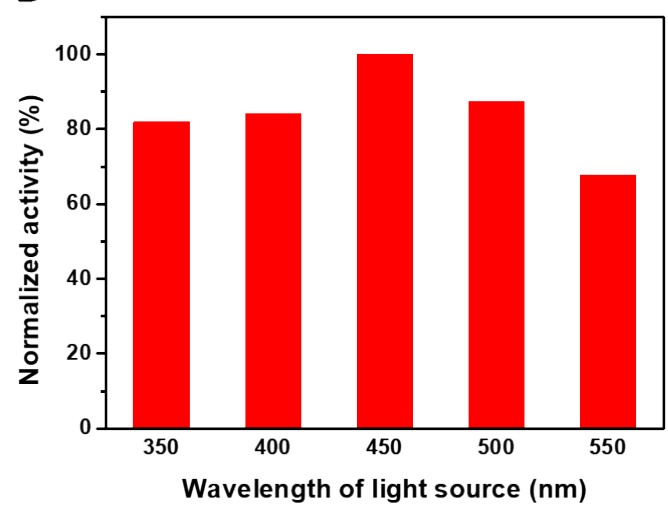

Figure S6. (A) UV-visible absorption spectra of the oxidized TMB. Five samples in $500 \mu \mathrm{L}$ acetate buffer $(0.10 \mathrm{M}, \mathrm{pH}$ 4.0) containing PSMOF ( $40 \mu \mathrm{L}, 3 \mathrm{mg} / \mathrm{mL})$ and TMB $(10 \mu \mathrm{L}, 25 \mathrm{mM})$ were irradiated under a compact light for $30 \mathrm{~min}$. (B) Normalized irradiation wavelength-dependent oxidase-mimicking activity of PSMOF.

A compact light (RF-5301PC spectrophotometer, Shimadzu, Japan) which can irradiate over a broad range of wavelengths was employed to measure the irradiation wavelength-dependent effects on the oxidase-like activity. As shown in Figure S6A, UV-visible absorption spectra of the oxidized TMB were collected under the irradiation of different wavelengths. Figure S6B showed the normalized irradiation wavelength-dependent oxidase-like activity. The wavelength of $450 \mathrm{~nm}$ produced the best oxidase-like activity while the wavelength of $550 \mathrm{~nm}$ produced the lowest activity, which was consistent with the absorption of the PSMOF. 


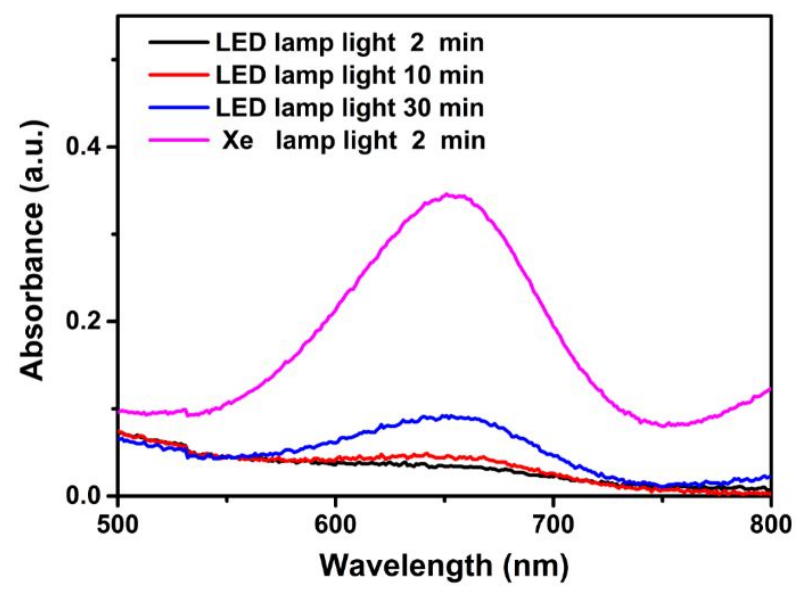

Figure S7. Effects of light source on the oxidase-mimicking activity of PSMOF.

As shown in Figure S7, with the extension of the irradiation time from 2 to $30 \mathrm{~min}$, a LED lamp can drive the photocatalysis of the PSMOF. However, compared with a Xe lamp $(300 \mathrm{~W})$, the lower power $(27 \mathrm{~W})$ of the LED lamp produced much weaker oxidase-mimicking activity in 2 min of irradiation, suggesting that a Xe lamp was more suitable as the light source for efficiently driving the photocatalysis of the PSMOF. 
A

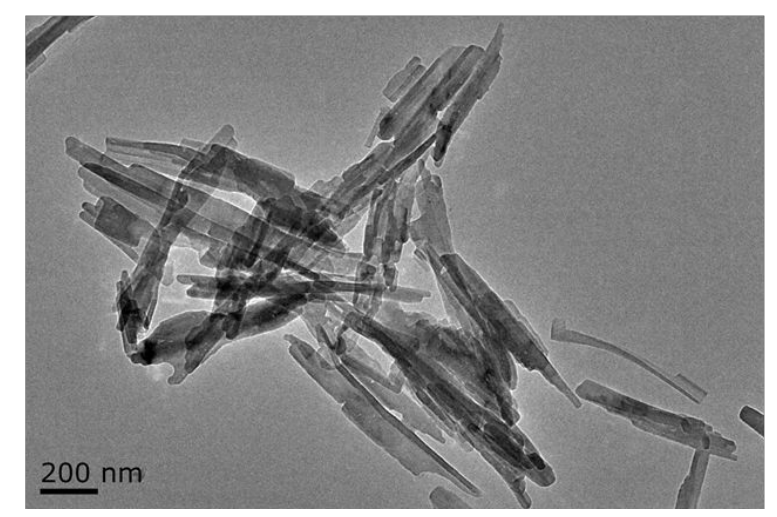

B

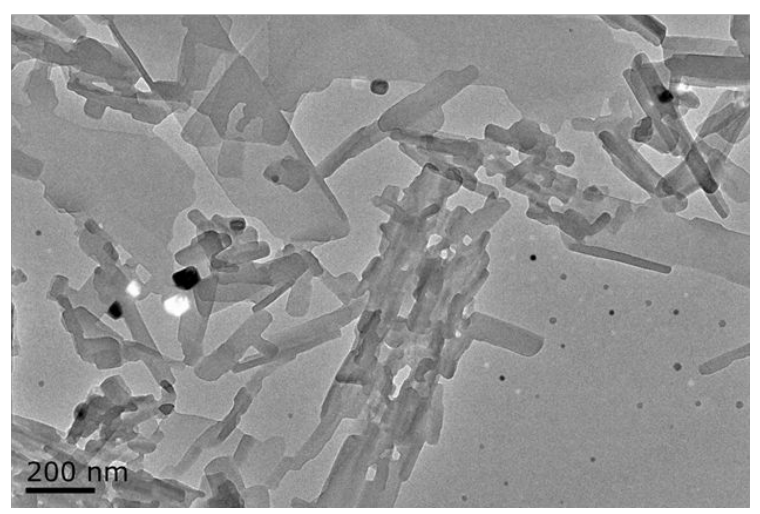

Figure S8. (A) TEM image of PSMOF after the catalytic reaction. (B) TEM image of PSMOF after 2 days of storage under $0.10 \mathrm{M}$ acetate buffer ( $\mathrm{pH} 4.0)$.

As shown in Figure S8A, after the catalytic reaction, the PSMOF showed no significant morphology changes, indicating that the PSMOF was stable under the catalytic conditions. However, after 2 days of storage in $0.10 \mathrm{M}$ acetate buffer $(\mathrm{pH}$ 4.0), the aggregation and degradation of the PSMOF was observed, suggesting the effect of proton on the coordination with $\mathrm{Zn}^{2+}$ (Figure S8B). 
A

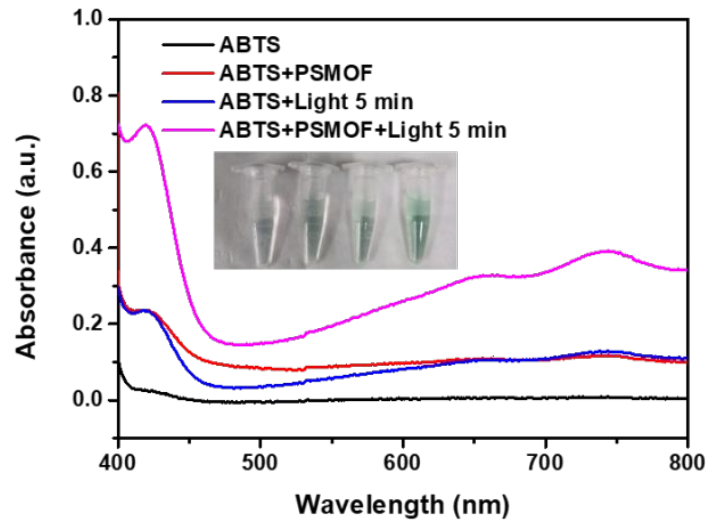

B

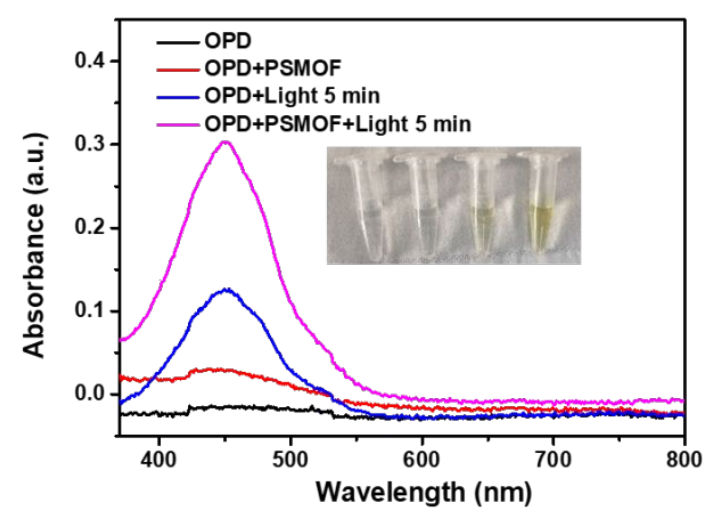

Figure S9. (A) UV-visible absorption spectra of four samples in $0.10 \mathrm{M}$ acetate buffer (pH 4.0) containing ABTS (no light irradiation), PSMOF+ABTS (no light irradiation), ABTS under light irradiation, and PSMOF+ABTS (under light irradiation). Inset: corresponding photograph of the four samples. (B) UV-visible absorption spectra of four samples in $0.10 \mathrm{M}$ acetate buffer ( $\mathrm{pH} 4.0$ ) containing OPD (no light irradiation), PSMOF+OPD (no light irradiation), OPD under light irradiation, and PSMOF+OPD under light irradiation. Inset: corresponding photograph of the four samples. 


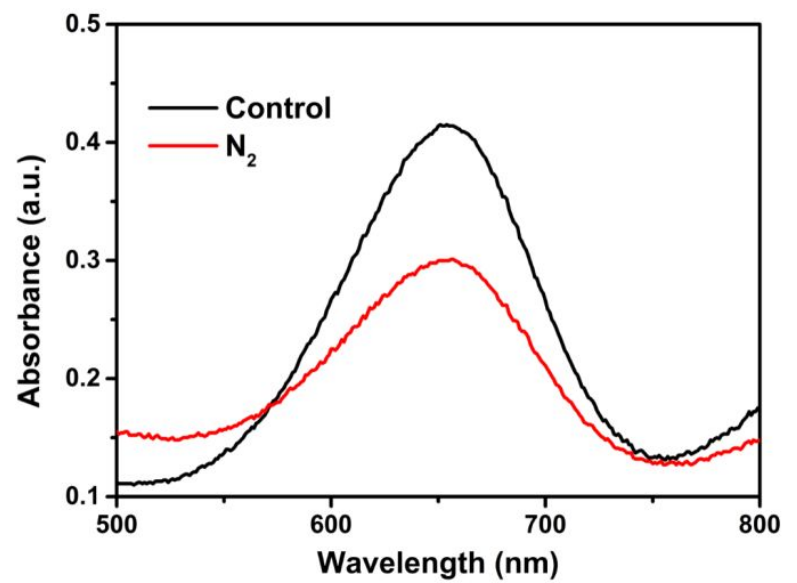

Figure S10. The effect of nitrogen on the oxidase-like catalytic activity of PSMOF.

The effect of oxygen on the activity of PSMOF for oxidizing TMB was investigated as follow. As shown in Figure S10, the catalytic activity of PSMOF decreased obviously in the nitrogen-rich atmosphere, implying the crucial role of the dissolved oxygen in the catalytic reaction. 


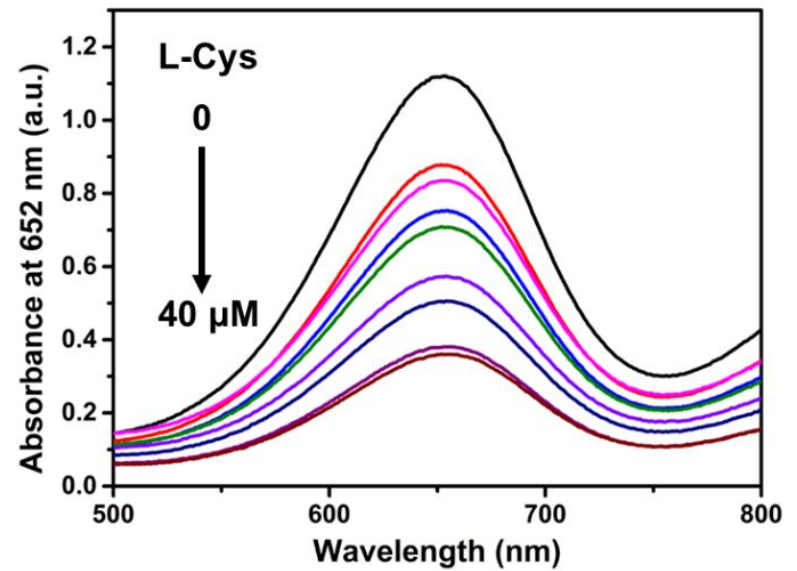

Figure S11. UV-visible absorption spectra of TMB after catalytic oxidation with PSMOF in the absence and presence of L-Cys with different concentrations $(0-40 \mu \mathrm{M})$. 


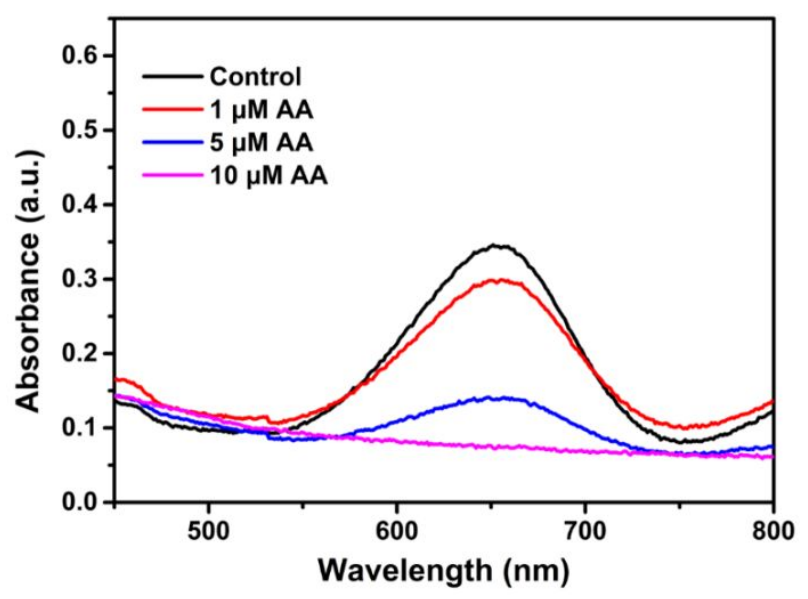

Figure S12. Effect of ascorbic acid on the oxidase-mimicking activity of PSMOF.

Figure S12 showed that the strong antioxidation activity of ascorbic acid (AA) inhibited the oxidase-mimicking activity of PSMOF, indicating that the PSMOF did not have the selectivity toward strong antioxidants. However, the intracellular AA was depleted over $96 \%$ during cell culture, so the presence of AA would not affect the detection of cellular GSH. 
A

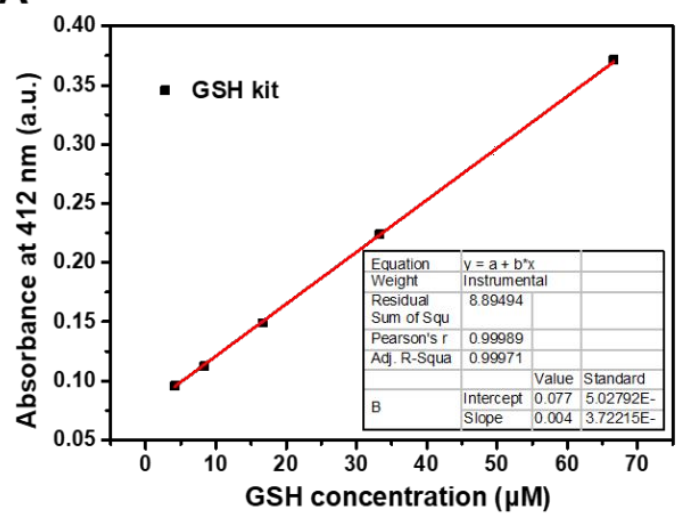

B

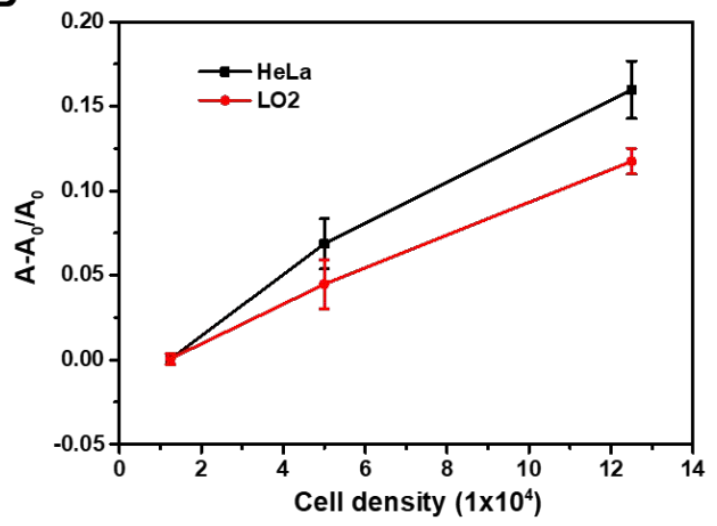

Figure S13. (A) Calibration curve of the commercial GSH kit in the concentration range from 4 to $66 \mu \mathrm{M}$. (B) GSH detection in different cell densities with the commercial GSH kit. The plot of $\left(\mathrm{A}-\mathrm{A}_{0}\right) / \mathrm{A}_{0}$ versus the cell density. $\mathrm{A}$, absorbance at $412 \mathrm{~nm}$ in the presence of cells. $\mathrm{A}_{0}$, absorbance at $412 \mathrm{~nm}$ in the absence of cells. Each error bar shows the standard deviation of three independent measurements.

The commercial GSH kit is based on the thiol-disulfide exchange reaction occurring between a thiol and [5,5-ditholbis(2nitrobenzoic acid), DTNB]. Thiols react with DTNB to form 2-nitro-5-thiobenzoate, which can be quantified by measuring the absorbance at $412 \mathrm{~nm}$. 
Table S1. Kinetics parameters of PSMOF nanozymes as well as laccase.

\begin{tabular}{|c|c|c|c|c|}
\hline Catalyst & Substrate & $\boldsymbol{K}_{\boldsymbol{m}}(\mathbf{m M})$ & $\boldsymbol{v}_{\boldsymbol{m a x}}\left(\mathbf{M s}^{-\mathbf{1}}\right)$ & Ref. \\
\hline PSMOF & TMB & 0.165 & $1.39 \times 10^{-7}$ & This work \\
\hline Laccase & TMB & 0.194 & $4.53 \times 10^{-8}$ & {$[1]$} \\
\hline
\end{tabular}


Table S2. Comparison of different methods for GSH detection.

\begin{tabular}{|c|c|c|c|c|}
\hline Methods & Materials & Linear range & LOD & Reference \\
\hline Colorimetry & Au nanoclusters & $2-25 \mu \mathrm{M}$ & $0.42 \mu \mathrm{M}$ & {$[2]$} \\
\hline Colorimetry & $\mathrm{MnO}_{2}$ nanosheets & $0.5-10 \mu \mathrm{M}$ & $0.1 \mu \mathrm{M}$ & {$[3]$} \\
\hline Colorimetry & $\mathrm{Fe}_{3} \mathrm{O}_{4} \mathrm{NPs}$ & $3.0-30 \mu \mathrm{M}$ & $3 \mu \mathrm{M}$ & {$[4]$} \\
\hline Fluorimetry & Carbon dots- $\mathrm{MnO}_{2}$ & $1-10 \mu \mathrm{M}$ & $0.3 \mu \mathrm{M}$ & {$[5]$} \\
\hline Fluorimetry & Quantum-dot & $5-250 \mu \mathrm{M}$ & $0.6 \mu \mathrm{M}$ & {$[6]$} \\
\hline Fluorimetry & $\begin{array}{l}\text { BODIPY-based } \\
\text { fluorescent probe }\end{array}$ & $12.5-200 \mu \mathrm{M}$ & $0.85 \mu \mathrm{M}$ & {$[7]$} \\
\hline Electrochemistry & $\begin{array}{c}\mathrm{CdSe} / \mathrm{ZnS} \text { quantum } \\
\text { dots }\end{array}$ & $10-180 \mu \mathrm{M}$ & $1.5 \mu \mathrm{M}$ & {$[8]$} \\
\hline SERS & Au nanoflowers & $1.1-23.5 \mu \mathrm{M}$ & $0.4 \mu \mathrm{M}$ & [9] \\
\hline Colorimetry & PSMOF & $1-20 \mu \mathrm{M}$ & $0.68 \mu \mathrm{M}$ & This work \\
\hline
\end{tabular}

LOD: limit of detection.

\section{References.}

(1) Liang, H.; Lin, F.; Zhang, Z.; Liu, B.; Jiang, S.; Yuan, Q.; Liu, J. ACS Appl. Mater. Interfaces 2017, 9, $1352-1360$.

(2) Feng, J.; Huang, P.; Shi, S.; Deng, K.-Y.; Wu, F.-Y. Anal. Chim. Acta 2017, 967, 64-69.

(3) Huang, Z.-M.; Cai, Q.-Y.; Ding, D.-C.; Ge, J.; Hu, Y.-L.; Yang, J.; Zhang, L.; Li, Z.-H. Sens. Actuators, B 2017, 242 , 355-361.

(4) Ma, Y.; Zhang, Z.; Ren, C.; Liu, G.; Chen, X. Analyst 2012, 137, 485-489.

(5) Cai, Q.-Y.; Li, J.; Ge, J.; Zhang, L.; Hu, Y.-L.; Li, Z.-H.; Qu, L.-B. Biosens. Bioelectron. 2015, 72, 31-36.

(6) Liu, J.; Bao, C.; Zhong, X.; Zhao, C.; Zhu, L. Chem. Commun. 2010, 46, 2971-2973.

(7) Wang, F.; Zhou, L.; Zhao, C.; Wang, R.; Fei, Q.; Luo, S.; Guo, Z.; Tian, H.; Zhu, W.-H. Chemi. Sci. 2015, 6, 25842589.

(8) Dennany, L.; Gerlach, M.; O'Carroll, S.; Keyes, T. E.; Forster, R. J.; Bertoncello, P. J. Mater. Chem. 2011, 21 , 1398413990.

(9) Wang, W.; Zhang, L.; Li, L.; Tian, Y. Anal. Chem. 2016, 88, 9518-9523. 